\title{
A Sensitive HPLC-MS/MS Analysis of Dencichine in Rat Plasma and Its Application to Pharmacokinetics
}

\author{
Chen Qian ${ }^{1}$, Yanjuan Yuan ${ }^{2}$, Xuejun $\mathrm{He}^{1}$, Jing Liu ${ }^{2}$, Qing Shao ${ }^{2}, \mathrm{Hua} \mathrm{Wu}^{2}$, Hongqun Qiao ${ }^{3 *}$ \\ ${ }^{1}$ School of Biotechnology and Pharmaceutical Engineering, Nanjing University of Technology, Nanjing, China \\ ${ }^{2}$ Jiangsu Center for Safety Evaluation of Drugs, Nanjing, China \\ ${ }^{3}$ School of Pharmaceutical Sciences, Nanjing University of Technology, Nanjing, China \\ Email: "qiaohongqun@sina.com
}

Received May 21, 2012; revised June 24, 2012; accepted July 12, 2012

\begin{abstract}
In order to quantitate dencichine in biological samples, a selective and sensitive method for the determination of dencichine in rat plasma based on high-performance liquid chromatography-tandem mass spectrometry (HPLC-MS/MS) was developed and validated. (1)-2-amino-3-(carboxymethylthio)propionic acid was used as the internal standard (I.S.). After a protein precipitation extraction with acetonitrile, dencichine and the I.S. were chromatographed on an Xterra MS-C18 column. The mobile phase was consisted of $20 \mathrm{mmol} / \mathrm{L}$ ammonium acetate solution-acetonitrile $(35: 65, \mathrm{~V} / \mathrm{V})$ at a flow rate of $0.2 \mathrm{~mL} / \mathrm{min}$. The detection was performed on a triple quadrupole mass via electrospray ionization (ESI) source in the positive mode. The intra- and inter-day precision (relative standard deviation, R.S.D.) values of dencichine were below $6.7 \%$. The extraction recoveries were up $85 \%$. The lower limit of quantification was $20 \mathrm{ng} / \mathrm{ml}$, which was sensitive enough to detect the analyte. The HPLC-MS/MS method was successfully applied to the pharmacokinetic study after an intravenous administration of dencichine in Sprague-Dawley (SD) rat.
\end{abstract}

Keywords: Dencichine; (L)-2-Amino-3-(Carboxymethylthio)Propionic Acid; Hplc-Ms/Ms; Pharmacokinetic; Ammonium Acetate Solution-Acetonitrile (35:65, V/V) Mobile Phase

\section{Introduction}

Dencichine ( $\beta$ - $N$-oxalyl-1- $\alpha, \beta$-diaminopropionic acid) (Figure 1) is a non-protein amino acid which is isolated from the seeds of Lathyrus sativus. And it is also contained in other plants, including other genera of leguminous and non-leguminous plants, such as Panax ginseng, Panax notoginseng and Panax quinquefolius [1-3]. It has been found that in many modes of bleeding, dencichine has obviously preventive and therapeutic properties of hemostasis [4]. Shortening the time of hemostasis, promoting the blood clotting, reducing plasma tPA level and thus decreasing Fibrinolytic activity can directly account for the hemostatic function. In addition, dencichine has been proven to be a kind of neurotoxins [5]. It was also discovered that the hemostasis effect happens at a low dose of dencichine, while the neurotoxins occurs at a high dose [6,7]. So in order to develop the dencichine as a potential and safe hemostatic agent in clinical treatment, the preclinical pharmacokinetic study is desirable. In this research, a sensitive method to quantificate dencichine in plasma samples was developed and validated and it was successfully applied to a preclinical pharmacokinetic

${ }^{*}$ Corresponding author. study.

There are few published reports about the determination of dencichine in biological samples. Most of the previous analytical methods were developed for the determination of dencichine in food or seed samples [8-13]. And the recent reports about the determination of dencichine were all using the method of derivatization $[4,14$, 15]. As is known to all, derivation was an effective way to enhance signal intensity. Meanwhile, finding a suitable derivatization reagent is supposed to be a complicated procedure. Although previously, a liquid chromatography with tandem mass spectrometric method was used to determine dencichine in plant species without any derivatization [6], the LLOQ was $1.5 \mu \mathrm{g} / \mathrm{ml}$ and it was not sensitive enough to determine dencichine in plasma for the pharmacokinetic studies. Now, we optimized some methodological aspects like mobile phase and MS setting in order to achieve better sensitivity. Carbocisteine (99.6\%) (Figure 1) was used as the internal standard. We developed a high performance liquid chromatographyelectrospray ionization-tandem mass spectrometry method for the determination of dencichine in rat plasma without any derivatization. This study described a selective, rapid and sensitive HPLC-MS/MS method for the 
<smiles>NC(CNC(=O)C(=O)O)C(=O)O</smiles>

(a)<smiles>NC(CSCC(=O)O)C(=O)O</smiles>

(b)

Figure 1. The chemical structure of dencichine (a) and carbocisteine (b).

quantitative determination of dencichine in rat plasma. The sample preparation was simple involving one-step precipitation. The total run time about $1.7 \mathrm{~min}$ and the simple composition of the mobile phase made the method attractive particularly in analyzing large number of plasma samples for pharmacokinetic study. In addition, this method avoided using derivatization which was timeconsuming, at the same time, it also met the requirement of sensitivity with the LLOQ of $20 \mathrm{ng} / \mathrm{ml}$. So this method could be successfully applied to the pharmacokinetic study after intravenous administration of dencichine in rat.

\section{Materials and Methods}

\subsection{Chemicals and Reagents}

Dencichine (100.27\%) was kindly provided by Jiangsu center for safety evaluation of drugs. Carbocisteine (99.6\%) was purchased from Tokyo Kasei Kogyo Co. Ltd (TCI). Acetonitrile and ammonium acetate were purchased from TEDIA Corporation of America and both of them were of HPLC grade. Aqueous ammonia was of HPLC grade and purchased from ROE Scientific INC Corporation of America. The deionized water was used through the experiment. All other reagents used in the experiment were of analytical grade.

\subsection{Animals}

The animal experimental protocol was approved by the ethic committee of Jiangsu center for safety evaluation of drugs on March $5^{\text {th }}, 2010$. All animal studies were carried out according to the Guild for Care and Use of Laboratory Animals.

SD rats (half males and half females), weighing $250 \mathrm{~g}$ - $320 \mathrm{~g}$, were provided by Shanghai Slac Laboratory Animal CO. LTD.

\subsection{Analytical Methods}

Chromatographic analysis was performed on Waters 2695
LC system equipped with on-line vacuum degasser unit, a Quaternary pump and an autosampler. Mass spectrometric detection was performed on Waters Micro triple quadrupole mass spectrometer equipped with an ESI ion source.

LC separation was performed on Xterra MS-C18 column $(2.1 * 150 \mathrm{~mm}$ LD, $5 \mu \mathrm{m}$, Waters Corporation USA). The mobile phase was $20 \mathrm{mmol} / \mathrm{L}$ ammonium acetate and acetonitrile $(35: 65, \mathrm{v} / \mathrm{v})$ at a flow rate of 0.2 $\mathrm{ml} / \mathrm{min}$. The injection volume was $5 \mu \mathrm{L}$.

Mass spectrometric detection was performed in multiple reaction monitoring (MRM) mode with positive ionization mode. For analytes, the following optimized parameters were obtained: the capillary voltage was $3 \mathrm{KV}$, collision voltage was $10 \mathrm{~V}$, Gas Cell Parani was $3.2 \times$ $10^{-3}$ mbar, cone voltage was $20 \mathrm{~V}$, desolvation temperature was $350^{\circ} \mathrm{C}$, source temperature was $120^{\circ} \mathrm{C}$. Argon was used as collision gas, nitrogen was used as curtain gas $(50 \mathrm{~L} / \mathrm{h})$. Data processing was carried out with Masslynx 4.0 data analysis program. The precursor/product ion transitions selected were $\mathrm{m} / \mathrm{z} \quad 177.2 / 116.2$ for dencichine and $m / z$ 180.2/163.2 for I.S., respectively.

\subsection{Preparation of Stock Solutions}

Primary stock solutions of dencichine and I.S. with a concentration of $1 \mathrm{mg} / \mathrm{mL}$ were prepared by dissolving $10 \mathrm{mg}$ denecichine and I.S. with acetonitrile-aqueous ammonia $(1: 1, \mathrm{v} / \mathrm{v})$ solution. A series of working stock solutions $(0.1,0.25,0.5,1.0,2.5,5,10,25 \mu \mathrm{g} / \mathrm{mL})$ of dencichine were prepared by diluting of the primary stock solution with $20 \mathrm{mmol} / \mathrm{L}$ ammonium acetate solution: acetonitrile $(35: 65, \mathrm{v} / \mathrm{v})$. The working solution of I.S. was $6.25 \mu \mathrm{g} / \mathrm{mL}$ by diluting of the primary stock solution of I.S. with $20 \mathrm{mmol} / \mathrm{L}$ ammonium acetate solution: acetonitrile $(35: 65, \mathrm{v} / \mathrm{v})$. All standard solutions were stored at $4^{\circ} \mathrm{C}$ when not in use.

\subsection{Calibration Standards and Quality Control Samples}

The calibration standards of dencichine (20, 50, 100, 200, $500,1000,2000,5000 \mathrm{ng} / \mathrm{mL}$ ) were prepared by spiking $40 \mu \mathrm{L}$ of blank plasma with $10 \mu \mathrm{L}$ of working solution. Blank plasma samples (without I.S.) were also analyzed. The quality control (QC) samples of 50, 500, 2000 $\mathrm{ng} / \mathrm{mL}$ were prepared in the same way as calibration standards.

\subsection{Plasma Sample Preparation}

A $50 \mu \mathrm{L}$ aliquot of plasma in $2 \mathrm{~mL}$ centrifuge tube and $10 \mu \mathrm{L}$ of I.S. $(6.25 \mu \mathrm{g} / \mathrm{mL})$ were added. The mixture was vortex-mixed for $1 \mathrm{~min}$. Then the sample mixture was precipitated with $100 \mu \mathrm{L}$ acetonitrile in the tube and vortexmixed for another $5 \mathrm{~min}$. $120 \mu \mathrm{L}$ solution of upper layer 
was transferred into another $1.5 \mathrm{~mL}$ centrifuge tube after centrifugation at $15000 \mathrm{rmp}$ for $10 \mathrm{~min}$. Followed by another centrifugation at $15000 \mathrm{rmp}$ for $10 \mathrm{~min}, 80 \mu \mathrm{L}$ solution supernatant was transferred into an inject vial and a $5 \mu \mathrm{L}$ aliquot was injected into the HPLC-MS/MS system for analysis.

\subsection{Method Validation}

According to the technical guidelines of Clinical pharmacokinetics of chemicals, specificity, linearity, sensitivity, precision, accuracy, extraction recovery, matrix effects and stability were investigated to validate the method.

\subsubsection{Specificity}

By analyzing blank plasma samples, blank plasma samples with dencichine and I.S. and spiked plasma samples after an intravenous administration from six different sources and comparing their results, there should be no interference from endogenous substances which was contained in biological samples and the corresponding metabolites, degradation products.

\subsubsection{Linearity and LLOQ}

Calibration curve reflects the relationship between the concentration of analyte and instrument response value. In this study, it was evaluated by linear regression equation fitted with the peak area ratios of dencichine to I.S. against the dencichine concentration. The linear equation and the coefficient of determination were used to determine the linear quality. The lower limit of quantitation (LLOQ) was defined as the lowest concentration on the calibration curve.

\subsubsection{Extraction Recovery}

The extraction recovery was determined by analyzing five replicates of plasma at three QC levels of 50, 500, $2000 \mathrm{ng} / \mathrm{ml}$. The peak areas of analytes obtained in the plasma extracts spiked with dencichine and I.S. before the extraction and after the extraction were calculated. The recovery was the ratio of the peak areas after the extraction to the peak areas before the extraction.

\subsubsection{Matrix Effect}

Other components may cause some effects on the determination of the analyte other than analyte itself. In this research, matrix effect was used to test such effects. The matrix effect was evaluated at three QC levels of 50, 500, $2000 \mathrm{ng} / \mathrm{ml}$. Using blank plasma and mobile phase to dilute standard solution at the concentration of 50, 500, $2000 \mathrm{ng} / \mathrm{ml}$, respectively. R was defined as the peak areas of dencichine and I.S. with blank plasma. $\mathrm{Rr}$ was defined as the ratio of the peak area of dencichine and I.S. in standard solution. The ratio $(100 \times \mathrm{R} / \mathrm{Rr})$ was defined as the matrix effect.

\subsubsection{Precision and Accuracy}

The accuracy, intra-day and inter-day precision were evaluated by analyzing five replicates of plasma at three QC levels of 50, 500, $2000 \mathrm{ng} / \mathrm{ml}$ on three separate days. The precision was defined as the relative standard deviation (RSD) calculated from replicate measurement of QCs. RSD within $\pm 20 \%$ for the LLOQ and within $\pm 15 \%$ for the other concentration was desirable. Accuracy was defined as the percentage of comparing observed concentration to the nominal concentration.

\subsubsection{Stability}

The stability of dencichine in rat plasma was investigated at room temperature for $12 \mathrm{~h}$, at $-20^{\circ} \mathrm{C}$ for 42 days and after three freeze-thaw cycle at three QC levels of 50, $500,2000 \mathrm{ng} / \mathrm{ml}$. The concentrations of dencichine in these three different storage conditions were compared with the concentrations in the initial condition.

\subsection{Application to Pharmacokinetic Study}

Eighteen SD rats were divided into three groups, each group contained half male rats and half female rats. The rats were fasted before administration, but were free to access water. Rats were administrated dencichine, with a single dose of $1,5,25 \mathrm{mg} / \mathrm{ml}$ for three groups. After the administration, blood samples were collected in tubes at $0,5,15,30 \mathrm{~min}$ and 1, 2, 3, 4, 6, 8, 12, $24 \mathrm{~h}$, including the sample which was collected before administration. All blood samples were centrifuged at $4000 \mathrm{r} / \mathrm{min}$ for 10 min. Transferring the upper layers to another clean tubes for storage at $-20^{\circ} \mathrm{C}$ before use.

Pharmacokinetic parameters such as maximum plasma concentration (Cmax), time to reach the maximum concentration (Tmax), the area under the plasma concentration-time curve from zero to the time of the final measurable sample $\left(\mathrm{AUC}_{(0-24)}\right)$, the area under the plasma concentration-time curve from zero to infinity $\left(\mathrm{AUC}_{(0-\infty)}\right)$ were investigated by DAS2.1.1.

\section{Results}

\subsection{Optimization of HPLC-MS-MS Condition}

By optimizing the mobile phase and MS setting, we finally developed a simple and sensitive method to determine the concentration of dencichine. The retention time for dencichine and I.S. were both approximate $1.7 \mathrm{~min}$ utes. Multiple reaction monitoring (MRM) mode was performed to monitor precursor/product ion of $\mathrm{m} / \mathrm{z}$ 177.2/116.2 for dencichine and $m / z$ 180.2/163.2 for the I.S. (Figure 2). 

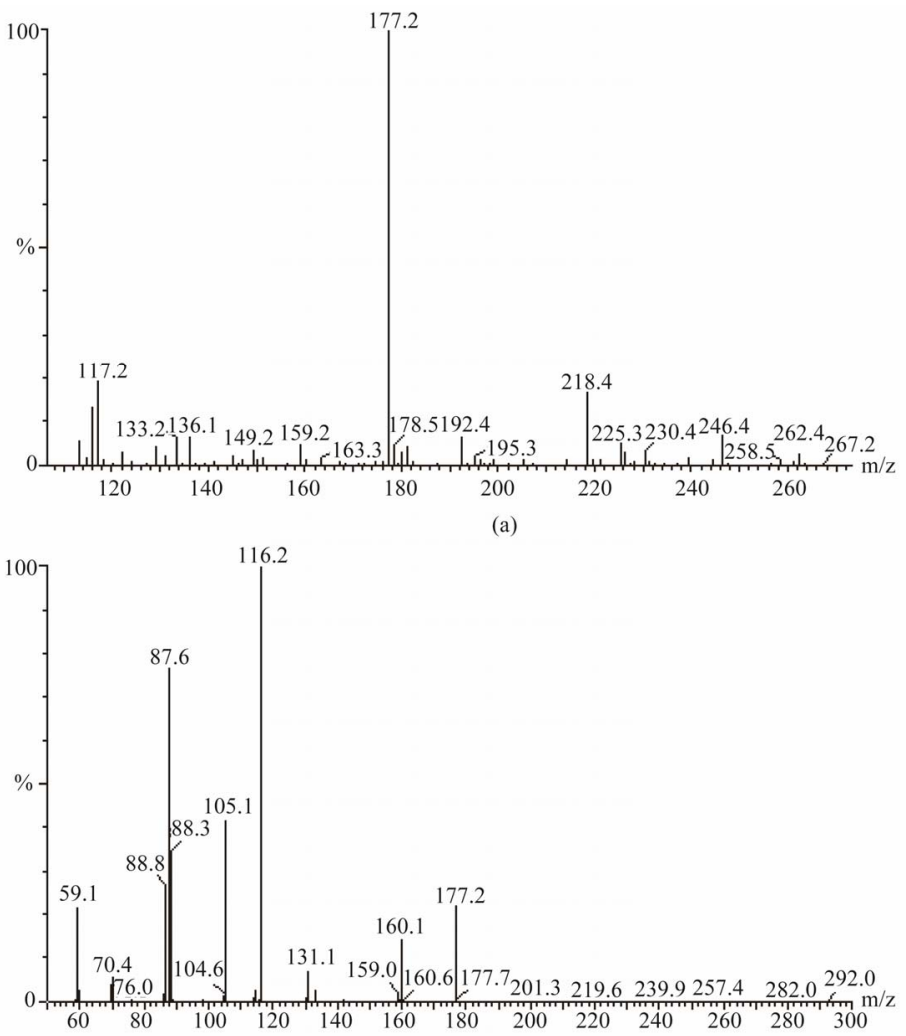

(b)

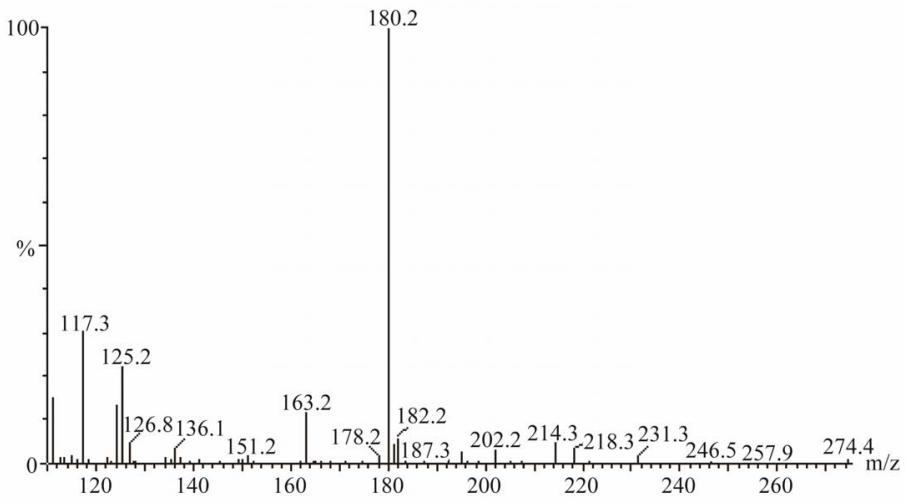

(c)

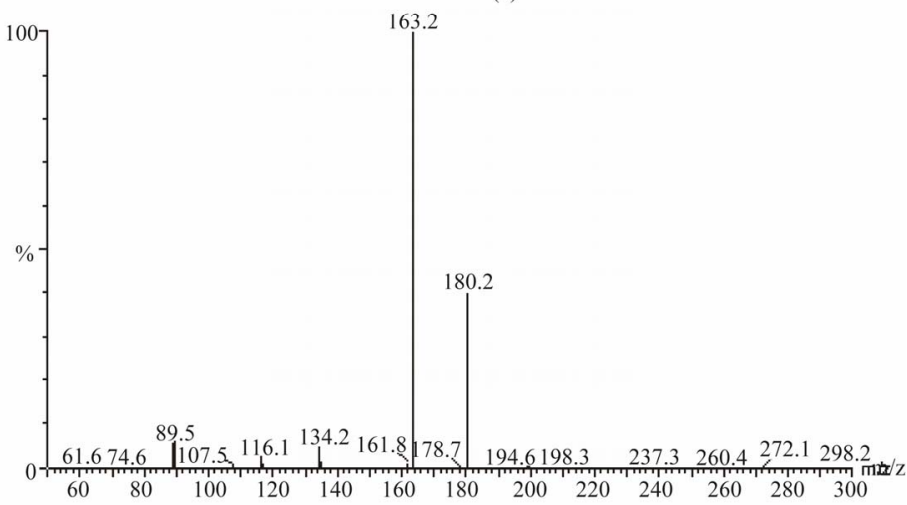

(d)

Figure 2. Full-scan mass spectra of dencichine (a); product-ion of dencichine (b); carbocysteine (c); product-ion of carbocysteine (d). 


\subsection{Sample Preparation}

The common extraction techniques include liquid-liquid extraction (LLE), Solid phase extraction (SPE) and protein precipitation. Since protein precipitation is considered to be the simplest way to extract samples, it was tried first and dencichine and I.S. can be easily detected with this method. LLE and SPE were also tested to compare with protein precipitation method in order to find out which method has the highest sensitivity. It was found that dencichine and I.S. were hardly to be detected with LLE and SPE. So protein precipitation was chosen finally.

\subsection{Method Validation}

\subsubsection{Specificity}

Representative MRM chromatograms of dencichine and I.S. in rat plasma samples are shown in Figure 3(a) blank plasma sample, (b) blank plasma sample spiked with dencichine and I.S., (c) spiked plasma samples after intravenous administration. The retention time of dencichine and I.S. were about $1.5 \mathrm{~min}$, respectively. And there was no interference signals detected within the retention time of dencichine and I.S.

\subsubsection{Linearity and LLOQ}

It was indicated that during the scope of $20-5000 \mathrm{ng} / \mathrm{ml}$, the response was good. A typical equal of the calibration curves we obtained was $\mathrm{y}=1.1053 \mathrm{E}-04 \mathrm{x}+0.0680\left(\mathrm{r}^{2}=\right.$ 0.9997).

It indicated that during the scope of $20-5000 \mathrm{ng} / \mathrm{ml}$, the response was good. The lower limit of quantitation was $20 \mathrm{ng} / \mathrm{ml}$ and it was sensitive enough to detect analyte.

\subsubsection{Extraction Recovery}

The results of extraction recovery were showed in Table 1 , at the concentration of $50,500,2000 \mathrm{ng} / \mathrm{ml}$, the mean extraction recoveries were $98.52 \%, 96.80 \%, 95.89 \%$, respectively. These results suggested that the recoveries were well within the acceptable limits and were not depending on the concentration.

\subsubsection{Matrix Effect}

In order to evaluate the matrix effect, I.S. at the concentration of $10 \mathrm{ug} / \mathrm{ml}$ and five lots of plasma samples at three different concentration levels $(50,500,2000 \mathrm{ng} / \mathrm{ml})$ were detected, and the results were shown in Table 1. The mean matrix effects of three concentrations were $97.56 \%, 99.01 \%, 98.51 \%$, respectively. These results indicated that no matrix effect was within the acceptable range.

\subsubsection{Precision and Accuracy}

QC plasma samples at the concentrations of 50, 500,
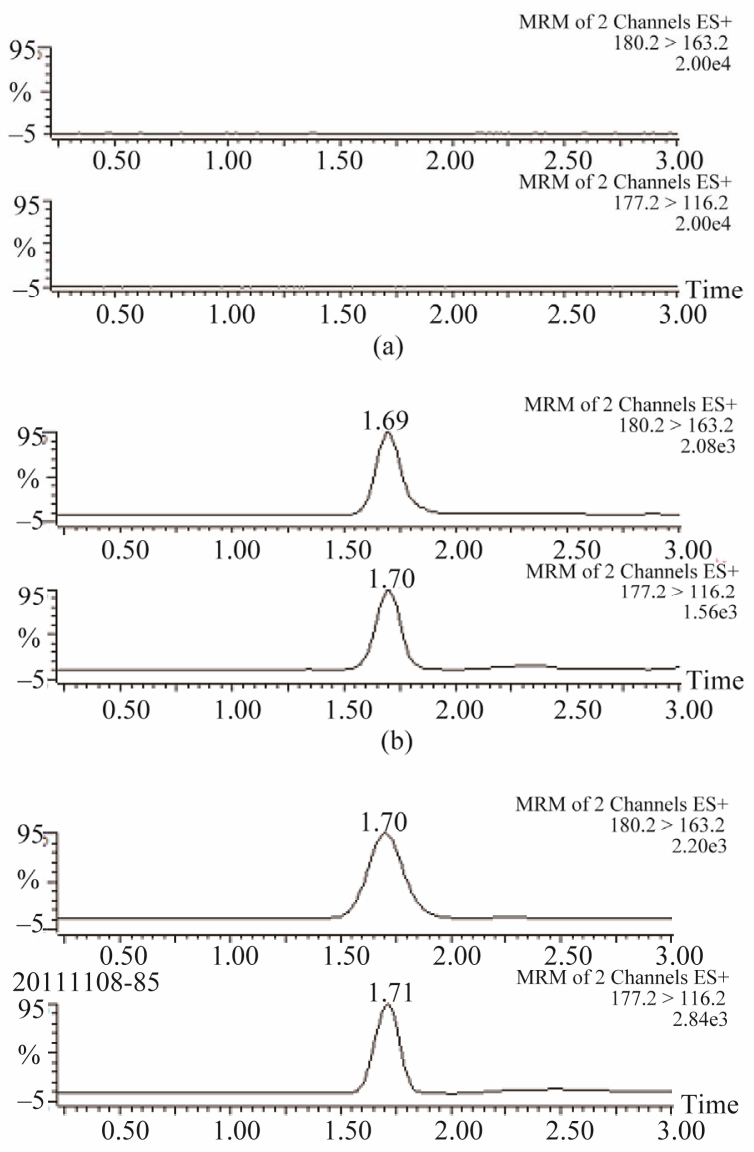

(c)

Figure 3. MRM chromatograms of (a) Blank plasma; (b) Blank sample added with dencichine $(500 \mathrm{ng} / \mathrm{ml})$ and I.S. $(10 \mathrm{ug} / \mathrm{ml})$; (c) A plasma sample from a rat $1 \mathrm{~h}$ after i.v. administration of $5 \mathrm{mg} / \mathrm{kg}$.

$2000 \mathrm{ng} / \mathrm{ml}$ were analyzed to evaluate the precision $(\mathrm{n}=$ 3 ) and accuracy. As the results showed in Table 2, the inter-day precision was less than $4.7 \%$, the intra-day precision was less than $4.8 \%$, these results were all within the acceptable limits which indicate that R.S.D. should be less than $15 \%$ and relative error should be within $\pm 15 \%$. So the method was proved to be precise and accurate.

\subsubsection{Stability}

As the results showed in Table 3, they indicated that no significant change occurred at three freeze-thaw cycles, long-term storage at $-20^{\circ} \mathrm{C}$ for 42 days, and room temperature for 12 hours, so all the investigated compounds were proved to be stable.

\subsection{Application in Pharmacokinetic Studies}

This method was successfully applied to investigate the pharmacokinetic studies of dencichine in rats plasma after i.v. administrations of $1,5,25 \mathrm{mg} / \mathrm{ml}$, respectively. 
Table 1. The extraction recovery and matrix effect of dencichine in rat plasma by LC-MS/MS method $(n=5)$.

\begin{tabular}{ccccc}
\hline Spiked concentration $(\mathrm{ng} / \mathrm{ml})$ & Extraction recovery (\%, mean) & R.S.D. (\%) & Matrix effect (\%, mean) & R.S.D. (\%) \\
\hline 50 & 98.52 & 3.6 & 97.56 & 4.4 \\
500 & 96.80 & 4.6 & 99.01 & 4.0 \\
2000 & 95.89 & 1.8 & 97.89 & 3.9 \\
\hline
\end{tabular}

Table 2. Precision and accuracy for determination of dencichine in rat plasma from the method validation, $(n=3$ days, 5 replicates per day).

\begin{tabular}{|c|c|c|c|c|}
\hline \multicolumn{2}{|c|}{ Concentration (ng/ml) } & \multirow{2}{*}{ Intra-day R.S.D. (\%) } & \multirow{2}{*}{ Inter-day R.S.D. (\%) } & \multirow{2}{*}{ Accuracy R.E. (\%) } \\
\hline Nominal concentration & Calculated concentration & & & \\
\hline 50 & 50.34 & 4.8 & 4.7 & 0.7 \\
\hline 500 & 487.25 & 3.2 & 3.5 & -2.5 \\
\hline 2000 & 1975.71 & 4.1 & 4.4 & -1.2 \\
\hline
\end{tabular}

Table 3. Stability of dencichine in rat plasma under different storage conditions $(n=3$ days).

\begin{tabular}{|c|c|c|c|}
\hline & \multicolumn{3}{|c|}{ Theoretical concentration $(\mathrm{ng} / \mathrm{ml})$} \\
\hline & 50 & 500 & 2000 \\
\hline \multicolumn{4}{|c|}{ (A) Three freeze-thaw cycles $(\mathrm{n}=3)$} \\
\hline Mean (ng/ml) & 53.11 & 518.09 & 1904.77 \\
\hline R.S.D. $(\%)$ & 1.7 & 2.0 & 4.8 \\
\hline R.E. $(\%)$ & 6.2 & 3.6 & -4.8 \\
\hline \multicolumn{4}{|c|}{ (B) Plasma samples at room temperature for 12 hours $(\mathrm{n}=3)$} \\
\hline Mean (ng/ml) & 52.12 & 513.08 & 1969.54 \\
\hline R.S.D. $(\%)$ & 1.2 & 1.5 & 5.7 \\
\hline R.E. $(\%)$ & 4.2 & 2.6 & -1.5 \\
\hline \multicolumn{4}{|c|}{ (C) Long-term storage at $-20^{\circ} \mathrm{C}$ for 42 days } \\
\hline Mean (ng/ml) & 50.92 & 507.30 & 2034.10 \\
\hline R.S.D. $(\%)$ & 1.7 & 1.4 & 4.1 \\
\hline R.E. (\%) & 1.8 & 1.4 & 1.7 \\
\hline
\end{tabular}

The main pharmacokinetic parameters were calculated as follows: the elimination half-time $\left(\mathrm{T}_{1 / 2}\right)$ of dencichine was $6.3 \pm 1.1,7.8 \pm 1.9$ and $7.2 \pm 1.4 \mathrm{~h}$. The area under the plasma concentration-time curve from $0 \mathrm{~h}$ to $24 \mathrm{~h}$ $\left(\mathrm{AUC}_{0-24}\right)$ were $3925.7 \pm 475.9,8521.3 \pm 973.6$ and $23371.9 \pm 3951.8 \mu \mathrm{g} / \mathrm{L} \cdot \mathrm{h}$, and the area under the plasma concentration-time curve from $0 \mathrm{~h}$ to infinity $\left(\mathrm{AUC}_{0-\infty}\right)$ were $41907.5 \pm 588.1,8892.5 \pm 1031.6$ and $25275.6 \pm$ $4769.9 \mu \mathrm{g} / \mathrm{L} \cdot \mathrm{h}$. The maximum plasma concentration $(\mathrm{Cmax})$ were $1922.3 \pm 180.86,7322.0 \pm 836.7$ and $31086.0 \pm 7075.3$.

\subsection{Discussion}

Dencichine is a nonprotein amino acid, which has two carboxyl groups. In the most research on quantitation of dencichine [4], the method of derivatization was applied to in order to solve the problem that the ion abundance was too weak to detect. However, compared with underivatization, an obvious disadvantage of derivatization was that, it was inevitable to bring some impurity into the system, which may lead to interference signals. For example, common derivating agent, such as HCL, would cause ion suppression for the residual HCL in the sample solution after derivatization [4]. In our experiment, we firstly tried to find a method without deravatization, at the same time, our method should meet the requirement of sensitivity. At the outset, both positive and negative modes were tried and we discovered that positive mode was more sensitive than the negative mode. In order to obtain the optimal mass response of the materials, every parameter was carefully investigated. Coupled with 
MRM mode, full scan product ion spectra of dencichine and I.S. was applied to this experiment. For dencichine, the precursor $\rightarrow$ product ion transition of $\mathrm{m} / \mathrm{z}$ was 177.2 $\rightarrow 116.2$ and for I.S. was $180.2 \rightarrow 163.2$ for I.S. (Figure 2).

As for HPLC, the mobile phase was considered to have some great influence to the separation. Common mobile phase always contains methanol or acetonitrile as the organic phase, but in this experiment, any rate of methanol or acetonitrile with water cannot obtain the satisfactory results. Then the second consideration was adding formic acid, which was considered as an additive to improve the response of analyte, especially in positive mode. However, we still failed to detect the analyte for the weak abundance. Finally, we decided to try some amine salt compound. According to adjusting the proportion of ammonium acetate constantly, we at last determined the appropriate mobile phase, $20 \mathrm{mmol} / \mathrm{L}$ ammonium acetate-acetonitrile $(35: 65, \mathrm{~V} / \mathrm{V})$ was finally used. Although this method brought $\mathrm{NH} 4+$ into the system, it was much easier to eluate than those residual derivating agents and caused less negative interference to the signals.

\section{Conclusion}

A rapid and sensitive method has been developed and validated for the quantification of dencichine. With approximate $1.7 \mathrm{~min}$ retention time of dencichine and I.S, $20 \mathrm{ng} / \mathrm{ml}$ LLOQ and one-step protein precipitation, this method was proved to be suitable and simple to investigate the pharmacokinetics study of dencichine.

\section{Declarations}

\subsection{Conflict of Interest}

The Author(s) declare(s) that they have no conflicts of interest to disclose.

\subsection{Funding Acknowledgements}

This research was financially supported by Jiangsu center for safety evaluation of drugs and School of Pharmaceutical Sciences, Nanjing University of Technology.

\section{REFERENCES}

[1] S. L. Rao, P. R. Adiga and P. S. Sarma, "The Isolation and Characterization of Beta-N-Oxalyl-L- $\alpha, \beta$-Diaminopropionic Acid: A Neurotoxin from the Seeds of Lathyrus Sativus," Biochemistry, Vol. 3, No. 3, 1964, pp. 432-436. doi:10.1021/bi00891a022

[2] M. Y. Quereshi, D. J. Pilbeam, C. S. Evans and E. A. Bell, "Neurolathyrogen, $\alpha$-Amino- $\beta$-Oxalylaminopropionic Acid in Legume Seeds," Phytochemistry, Vol. 16, No. 4, 1977, pp. 477-479. doi:10.1016/S0031-9422(00)94332-2

[3] Y. H. Kuo, F. Ikegami and F. Lambein, "Neuroactive and Other Free Amino Acids in Seed and Young Plants of Panax Ginseng," Phytochemistry, Vol. 62, No. 7, 2003, pp. 1087-1091. doi:10.1016/S0031-9422(02)00658-1

[4] Y. Zhang, X. Y. Chen, X. Y. Li and D. F. Zhong, "Development of a Liquid Chromatographic-Tandem Mass Spectrometric Method with Precolumn Derivatization for the Determination of Dencichine in Rat Plasma," Analytica Chimica Acta, Vol. 566, No. 2, 2006, pp. 200-206. doi:10.1016/j.aca.2006.03.011

[5] E. Rubenstein, "Biologic Effects of and Clinical Disorders Caused by Nonprotein Amino Acids," Medicine, Vol. 79, 2000, pp. 80-89.

[6] H. L. Koh, A. J. Lau and E. C. Y. Chan, "Hydrophilic Interaction Liquid Chromatography with Tandem Mass Spectrometry for the Determination of Underivatized Dencichine ( $\beta$-N-oxalyl-L- $\alpha, \beta$-diaminopropionic acid) in Panax Medicinal Plant Species," Rapid Communications in Mass Spectrometry, Vol. 19, No. 10, 2005, pp. 1237-1244. doi: $10.1002 / \mathrm{rcm} .1928$

[7] G. Q. Zhao and X. X. Wang, "Hemostatic Constituent of Sanchi (Panax Notoginseng)-Dencichine," Chinese Traditional and Herbal Drugs, 1986, pp. 17-34.

[8] S. L. Rao, "A Sensitive and Specific Colorimetric Method for the Determination of $\alpha, \beta$-Diaminopropionic Acid and the Lathyrus Sativus Neurotoxin," Analytical Biochemistry, Vol. 86, No. 2, 1987, pp. 386-396. doi:10.1016/0003-2697(78)90762-5

[9] A. Geda, C. J. Briggs and S. Venkataram, "Determination of the Neurolathyrogen Beta-N-Oxalyl-L- $\alpha, \beta$-Diaminopropionic Acid Using High-Performance Liquid- Chromatography with Fluorometric Detection," Journal of Chromatography A, Vol. 635, No. 2, 1993, pp. 338-341. doi:10.1016/0021-9673(93)80378-L

[10] J. K. Khan, N. Kebede, Y. H. Kuo, F. Lambein and A. Debruyn, "Analysis of the Neurotoxin Beta-Odap and Its Alpha-Isomer by Precolumn Derivatization with Phenylisothiocyanate," Analytical Biochemistry, Vol. 208, No. 2, 1993, pp. 237-240. doi:10.1006/abio.1993.1038

[11] F. Wang, X. Chen, Q. Chen, X. C. Qin and Z. X. Li, "Determination of Neurotoxin 3-N-Oxalyl-2,3-diaminopropionic Acid and Non-Protein Amino Acids in Lathyrus Sativus by Precolumn Derivatization with 1-Fluoro-2,4dinitrobenzene," Journal of Chromatography A, Vol. 883, No. 1-2, 2000, pp. 113-118. doi:10.1016/S0021-9673(00)00264-8

[12] X. Chen, F. Wang, Q. Chen, X. C. Qin and Z. X. Li, "Analysis of Neurotoxin 3-N-Oxalyl-L-2,3-diaminopropionic Acid and Its Alpha-Isomer in Lathyrus Sativus by High-Performance Liquid Chromatography with 6-Amino-quinolyl-N-hydroxysuccinimidyl Carbamate (AQC) Derivatization," Journal of Agricultural and Food Chemistry, Vol. 48, No. 8, 2000, pp. 3383-3386. doi:10.1021/jf000033y

[13] Z. Y. Yang, C. J. Jiao, Y. P. Wang, F. M. Li, Y. M. Liang and Z. X. Li, "A Method for the Sim $\mu$ Ltaneous Determination of Beta-ODAP, Alpha-ODAP, Homoarginine and Polyamines in Lathyrus Sativus by Liquid Chromatography Using a New Extraction Procedure," Analytica Chi- 
mica Acta, Vol. 534, No. 2, 2005, pp. 199-205. doi:10.1016/j.aca.2004.11.051

[14] G. X. Xie, Y. P. Qiu, M. F. Qiu, X. F. Gao, Y. M. Liu and W. Jia, "Analysis of Dencichine in Panax Notoginseng by Gas Chromatography-Mass Spectrometry with Ethyl
Chloroformate Derivatization," Journal of Pharmaceutical and Biomedical Analysis, Vol. 43, No. 3, 2007, pp. 920-925. doi:10.1016/j.jpba.2006.09.009

[15] G. D. Zhang, J. F. Cui and H. Y. Liu, Chinese Journal of Pharmaceutical Analysis, Vol. 10, 1990, pp. 209-212. 